UWThPh-1996-42

DFTT 34/96

hep-ph/9607372

July 1996

\title{
Neutrino mass spectrum from the results of neutrino oscillation experiments
}

\author{
S.M. Bilenky \\ Joint Institute for Nuclear Research, Dubna, Russia, and \\ INFN, Sezione di Torino, Via P. Giuria 1, I-10125 Torino, Italy \\ C. Giunti \\ INFN, Sezione di Torino, and Dipartimento di Fisica Teorica, Università di Torino, \\ Via P. Giuria 1, I-10125 Torino, Italy \\ and \\ W. Grimus \\ Institute for Theoretical Physics, University of Vienna, \\ Boltzmanngasse 5, A-1090 Vienna, Austria
}

\begin{abstract}
All the possible schemes of neutrino mixing with four massive neutrinos inspired by the existing experimental indications in favor of neutrino mixing are considered in a model independent way. Assuming that in short-baseline experiments only one mass-squared difference is relevant, it is shown that the scheme with a neutrino mass hierarchy is not compatible with the experimental results. Only two schemes with two pairs of neutrinos with close masses separated by a mass difference of the order of $1 \mathrm{eV}$ are in agreement with the results of all experiments. One of these schemes leads to possibly observable effects in ${ }^{3} \mathrm{H}$ and $(\beta \beta)_{0 \nu}$ experiments.
\end{abstract}




\section{Introduction}

Neutrino masses and neutrino mixing are natural and plausible phenomena of modern gauge theories (see, for example, Ref.[1]). However, for the time being, the values of the neutrino masses and mixing angles cannot be predicted on theoretical grounds. The determination of these quantities is the key problem of today's experimental neutrino physics.

At present there are several indications in favor of neutrino masses and mixing. One of the most important indications comes from solar neutrino experiments (Homestake [2], Kamiokande [3], GALLEX [4] and SAGE [5]). As it is well-known, in all four presently operating solar neutrino experiments the observed event rates are significantly smaller than the values predicted by the Standard Solar Model (SSM) [6]. Moreover, if the survival probability of solar $\nu_{e}$ 's is equal to one, the data of different experiments cannot be explained even if the total neutrino fluxes are considered as free parameters [7]. Assuming the validity of the SSM, the experimental data can be described by the MSW matter effect [8] for $\Delta m^{2} \sim 10^{-5} \mathrm{eV}^{2}$ [9] or by vacuum oscillations in the case of $\Delta m^{2} \sim 10^{-10} \mathrm{eV}^{2}$ 10] $\left(\Delta m^{2}\right.$ is the neutrino mass-squared difference).

The second indication in favor of neutrino mixing comes from the data of the Kamiokande [11], IMB [12] and Soudan [13] atmospheric neutrino experiments. The ratio of muon-like to electron-like events measured in these experiments is less than the expected ratio. The sub-GeV and multi-GeV data of the Kamiokande collaboration can be explained by $\nu_{\mu} \leftrightarrows \nu_{\tau}$ or $\nu_{\mu} \leftrightarrows \nu_{e}$ oscillations with $\Delta m^{2} \sim 10^{-2} \mathrm{eV}^{2}$

Finally, in the LSND experiment [14] $\bar{\nu}_{e} p \rightarrow e^{+} n$ events produced by neutrinos originating from $\pi^{+}$and subsequent $\mu^{+}$decays at rest were observed. These events can be explained by $\bar{\nu}_{\mu} \leftrightarrows \bar{\nu}_{e}$ oscillations with $\Delta m^{2} \sim 1 \mathrm{eV}^{2}$

Therefore, from the existing experimental data we have three different indications in favor of non-zero neutrino masses, each with a particular scale of $\Delta m^{2}$. However, we must also take into account the fact that in several short-baseline experiments neutrino oscillations were not observed. The resuls of these experiments allow to exclude large regions in the space of the neutrino oscillation parameters.

In the present paper we will examine what information on the neutrino mass spectrum can be inferred from the results of all short-baseline neutrino oscillation experiments if we also take into account the results of the solar and atmospheric neutrino experiments. We will present a general discussion which does not assume a definite model of neutrino mixing. We will show that the experimental results favor two rather particular possibilities for the neutrino mass spectrum.

We will start with the presentation of the general formulas for the neutrino transition probabilities in short-baseline neutrino oscillation experiments (for details see Ref.[15]). Our basic assumption is that only one neutrino mass-squared difference is relevant for short-baseline neutrino oscillations. This assumption means that the neutrino mass spectrum consists of two groups of close masses, separated by a mass difference in the eV range. Denoting the neutrinos of the two groups by $\nu_{1}, \ldots, \nu_{r}$ and $\nu_{r+1}, \ldots, \nu_{n}$, respectively, with masses $m_{1} \leq \ldots m_{r}$ and $m_{r+1} \leq \ldots \leq m_{n}$, we can quantify our assumption by

$$
\frac{\Delta m_{i 1}^{2} L}{2 p} \ll 1 \quad \text { for } \quad i \leq r \quad \text { and } \quad \frac{\Delta m_{n i}^{2} L}{2 p} \ll 1 \quad \text { for } \quad i \geq r+1
$$


where $\Delta m_{i j}^{2} \equiv m_{i}^{2}-m_{j}^{2}, L$ is the distance between the neutrino source and detector and $p$ is the neutrino momentum. We would like to emphasize that the inequalities (11) are well satisfied for short-baseline experiments with the mass squared differences relevant for the explanation of the solar and atmospheric neutrino experiments.

Under the assumption of validity of the inequalities (11), the amplitude of the transition $\nu_{\alpha} \rightarrow \nu_{\beta}$ is given by

$$
\mathcal{A}_{\nu_{\alpha} \rightarrow \nu_{\beta}} \simeq \mathrm{e}^{-i E_{1} t}\left\{\delta_{\alpha \beta}+\sum_{i \geq r+1} U_{\beta i} U_{\alpha i}^{*}\left[\exp \left(-i \frac{\Delta m^{2} L}{2 p}\right)-1\right]\right\}
$$

Here $\Delta m^{2} \equiv m_{n}^{2}-m_{1}^{2}, U$ is the unitary $n \times n$ mixing matrix and $\nu_{\alpha}, \nu_{\beta}$ are any active or sterile neutrinos. (Note that the number of sterile states is $n-3$.) From Eq.(2), for the probability of the transition $\nu_{\alpha} \rightarrow \nu_{\beta}(\alpha \neq \beta)$ we obtain

$$
P_{\nu_{\alpha} \rightarrow \nu_{\beta}}=\frac{1}{2} A_{\alpha ; \beta}\left(1-\cos \frac{\Delta m^{2} L}{2 p}\right),
$$

where the oscillation amplitude $A_{\alpha ; \beta}$ is given by

$$
A_{\alpha ; \beta}=4\left|\sum_{i \geq r+1} U_{\beta i} U_{\alpha i}^{*}\right|^{2}=A_{\beta ; \alpha} .
$$

The survival probability of $\nu_{\alpha}$ is calculated as

$$
P_{\nu_{\alpha} \rightarrow \nu_{\alpha}}=1-\sum_{\beta \neq \alpha} P_{\nu_{\alpha} \rightarrow \nu_{\beta}}=1-\frac{1}{2} B_{\alpha ; \alpha}\left(1-\cos \frac{\Delta m^{2} L}{2 p}\right),
$$

where

$$
B_{\alpha ; \alpha}=\sum_{\beta \neq \alpha} A_{\alpha ; \beta}=4 \sum_{i \geq r+1}\left|U_{\alpha i}\right|^{2}\left(1-\sum_{i \geq r+1}\left|U_{\alpha i}\right|^{2}\right)
$$

Using the unitarity of the mixing matrix, from Eqs.(4) and (6) we have also

$$
\begin{aligned}
& A_{\alpha ; \beta}=4\left|\sum_{i \leq r} U_{\beta i} U_{\alpha i}^{*}\right|^{2}, \\
& B_{\alpha ; \alpha}=4 \sum_{i \leq r}\left|U_{\alpha i}\right|^{2}\left(1-\sum_{i \leq r}\left|U_{\alpha i}\right|^{2}\right) .
\end{aligned}
$$

The expressions (3)-(8) describe the transitions between all possible neutrino states, whether active or sterile. Let us stress that in the scheme under consideration the oscillations in all channels are characterized by the same oscillation length $L_{\mathrm{osc}}=4 \pi p / \Delta \mathrm{m}^{2}$. 


\section{Schemes with three massive neutrinos}

Neutrino oscillations in the scheme with three massive neutrinos and a mass hierarchy were considered in many papers [16, 17, 18]. In Ref. [17] it was shown that the results of the LSND experiment are compatible with the data of all the other experiments on the search for neutrino oscillations and the data of solar neutrino experiments only if the element $\left|U_{\mu 3}\right|$ of the neutrino mixing matrix is large (close to one) and $\left|U_{e 3}\right|,\left|U_{\tau 3}\right|$ are small.

In Refs. 18 the possibility was considered that the same $\Delta m^{2} \simeq 0.3 \mathrm{eV}^{2}$ is relevant for the LSND effect and for the atmospheric neutrino anomaly. This possibility seems to be rather marginal. In fact, from the results of the LSND [14 and Bugey [19] experiments it follows that the transition amplitude $A_{\mu ; e}$ is less than $4 \times 10^{-2}$. Therefore, in order to explain the sub-GeV Kamiokande data one needs a large amplitude $A_{\mu ; \tau} \simeq B_{\mu ; \mu}$. Taking into account the limits for $B_{\mu ; \mu}$ that follow from the CDHS $\nu_{\mu}$ disappearance experiment [20], one finds $\Delta m^{2} \lesssim 0.4 \mathrm{eV}^{2}$. On the other hand, the results of the LSND and Bugey experiments require $\Delta m^{2} \gtrsim 0.3 \mathrm{eV}^{2}$. Thus, the results of all short-baseline oscillation experiments are compatible with the Kamiokande sub-GeV data only if the value of $\Delta m^{2}$ lies in the very narrow interval $0.3 \mathrm{eV}^{2} \lesssim \Delta m^{2} \lesssim 0.4 \mathrm{eV}^{2}$ and $A_{\mu ; e} \simeq 3 \times 10^{-2}$. At such large values of $\Delta m^{2}$ the cosine in the expression (5) practically disappears for atmospheric neutrinos because of the averaging over energy and distance. This would mean that there cannot be an zenithal dependence of the double ratio $R=(\mu / e)_{\text {data }} /(\mu / e)_{\mathrm{MC}}$ of atmospheric muon and electron events $\left((\mu / e)_{\mathrm{MC}}\right.$ is the Monte-Carlo calculated ratio of muon and electron events without neutrino oscillations). However, some zenithal dependence of this double ratio is indicated by the multi-GeV Kamiokande data.

The existence of an zenithal dependence of the double ratio $R$ will be checked soon by the on-going Super-Kamiokande experiment 21]. Let us also mention that $\nu_{\mu} \leftrightarrows \nu_{\tau}$ oscillations with large amplitude and $\Delta m^{2} \simeq 0.3 \mathrm{eV}^{2}$ could be tested by CHORUS [22], NOMAD [23] and especially by the COSMOS [24] experiment.

Up to now we have discussed a scheme with three neutrinos and a mass hierarchy. The other possible scheme with three neutrinos is a scheme where the spectrum has the form $m_{1} \ll m_{2} \lesssim m_{3}$ [25]. With the same arguments as above it is possible to show [15] that this scheme is also disfavored by the experimental results.

\section{Four massive neutrinos with a mass hierarchy}

We will now assume that the anomaly in the sub-GeV and multi-GeV Kamiokande atmospheric neutrino data is due to neutrino oscillations, for which a scale of $\Delta m^{2} \sim 10^{-2} \mathrm{eV}^{2}$ is required [11]. In order to accomodate also the solar neutrino data and the LSND data, it is necessary to assume that the flavor neutrino fields are superpositions of (at least) four massive neutrino fields. We will consider now all possible schemes with four neutrinos and the dominance of one $\Delta m^{2}$ in short-baseline experiments. We will start with the case of a neutrino mass hierarchy, $m_{1} \ll m_{2} \ll m_{3} \ll m_{4}$, with $\Delta m_{21}^{2}$ and $\Delta m_{32}^{2}$ relevant for the suppression of the flux of solar neutrinos and for the atmospheric neutrino anomaly, respectively. This case corresponds to $n=4$ and $r=3$ in the formulas (3)-(8) and the 
oscillation amplitudes are given by

$$
\begin{aligned}
& A_{\alpha ; \beta}=4\left|U_{\beta 4}\right|^{2}\left|U_{\alpha 4}\right|^{2}, \\
& B_{\alpha ; \alpha}=4\left|U_{\alpha 4}\right|^{2}\left(1-\left|U_{\alpha 4}\right|^{2}\right) .
\end{aligned}
$$

We will consider the range $0.3 \mathrm{eV}^{2} \leq \Delta m^{2} \leq 10^{3} \mathrm{eV}^{2}$, which covers the sensitivity of all short-baseline experiments. At any fixed value of $\Delta m^{2}$, from the exclusion plots of the Bugey [19], CDHS [20] and CCFR [26] disappearance experiments we have

$$
B_{\alpha ; \alpha} \leq B_{\alpha ; \alpha}^{0} \quad(\alpha=e, \mu) .
$$

The values of $B_{e ; e}^{0}$ and $B_{\mu ; \mu}^{0}$ can be obtained from the corresponding exclusion curves. From Eqs.(10) and (11) we find that the elements $\left|U_{\alpha 4}\right|^{2}$ must satisfy one of the two inequalities

$$
\left|U_{\alpha 4}\right|^{2} \leq a_{\alpha}^{0} \quad \text { or } \quad\left|U_{\alpha 4}\right|^{2} \geq 1-a_{\alpha}^{0} \quad(\alpha=e, \mu)
$$

where (see Ref. [17])

$$
a_{\alpha}^{0}=\frac{1}{2}\left(1-\sqrt{1-B_{\alpha ; \alpha}^{0}}\right)
$$

In the range of $\Delta m^{2}$ considered here $a_{e}^{0}$ and $a_{\mu}^{0}$ are small $\left(a_{e}^{0} \lesssim 4 \times 10^{-2}, a_{\mu}^{0} \lesssim 10^{-1}\right)$. We will show now that solar neutrino data and the atmospheric neutrino anomaly exclude large values of $\left|U_{e 4}\right|^{2}$ and $\left|U_{\mu 4}\right|^{2}$. In fact, the average probability of solar neutrinos to survive is given by (see Refs.[27, 15])

$$
P_{\nu_{e} \rightarrow \nu_{e}}^{\odot}=\sum_{i=3,4}\left|U_{e i}\right|^{4}+\left(1-\sum_{i=3,4}\left|U_{e i}\right|^{2}\right)^{2} P_{\nu_{e} \rightarrow \nu_{e}}^{(1 ; 2)}
$$

where $P_{\nu_{e} \rightarrow \nu_{e}}^{(1 ; 2)}$ is the $\nu_{e}$ survival probability due to the mixing of $\nu_{e}$ with $\nu_{1}$ and $\nu_{2}$. If $\left|U_{e 4}\right|^{2} \geq 1-a_{e}^{0}$, from (14) we have $P_{\nu_{e} \rightarrow \nu_{e}}^{\odot} \gtrsim 0.92$ for all solar neutrino energies. Such a large lower bound is not compatible with the solar neutrino data.

The average probability of atmospheric $\nu_{\mu}$ 's to survive is given by

$$
P_{\nu_{\mu} \rightarrow \nu_{\mu}}^{\mathrm{atm}}=\left|U_{\mu 4}\right|^{4}+\left(1-\left|U_{\mu 4}\right|^{2}\right)^{2} P_{\nu_{\mu} \rightarrow \nu_{\mu}}^{(1,2 ; 3)}
$$

where $P_{\nu_{\mu} \rightarrow \nu_{\mu}}^{(1,2 ; 3)}$ is the $\nu_{\mu}$ survival probability due to the mixing of $\nu_{\mu}$ with $\nu_{3}$ and $\nu_{2}, \nu_{1}$. The double ratio of atmospheric muon and electron events is given by

$$
R=\frac{P_{\nu_{\mu} \rightarrow \nu_{\mu}}^{\mathrm{atm}}+r^{-1} P_{\nu_{e} \rightarrow \nu_{\mu}}^{\mathrm{atm}}}{P_{\nu_{e} \rightarrow \nu_{e}}^{\mathrm{atm}}+r P_{\nu_{\mu} \rightarrow \nu_{e}}^{\mathrm{atm}}}
$$

${ }^{1} P_{\nu_{\mu} \rightarrow \nu_{\mu}}^{(1,2 ; 3)}$ is given by

$$
P_{\nu_{\mu} \rightarrow \nu_{\mu}}^{(1,2 ; 3)}=1-2 \frac{\left(\left|U_{\mu 1}\right|^{2}+\left|U_{\mu 2}\right|^{2}\right)\left|U_{\mu 3}\right|^{2}}{\left(\left|U_{\mu 1}\right|^{2}+\left|U_{\mu 2}\right|^{2}+\left|U_{\mu 3}\right|^{2}\right)^{2}}\left(1-\cos \frac{\Delta m_{32}^{2} L}{2 p}\right) .
$$


where $r$ is the ratio of muon and electron events calculated without neutrino oscillations. For the Kamiokande sub-GeV events $r \simeq 1.57$ and there is no zenithal dependence of the double ratio $R$ [11]. This means that the oscillatory terms in the probabilities $P_{\nu_{\alpha} \rightarrow \nu_{\beta}}^{\text {atm }}$ $(\alpha, \beta=e, \mu)$ in Eq.(16) disappear because of the averaging over energy and distance. In this case $P_{\nu_{e} \rightarrow \nu_{\mu}}^{\mathrm{atm}}=P_{\nu_{\mu} \rightarrow \nu_{e}}^{\mathrm{atm}} \leq 1-P_{\nu_{\mu} \rightarrow \nu_{\mu}}^{\mathrm{atm}}$. From Eq.(16), we obtain for $R$ the lower bound

$$
R^{\min }=P_{\nu_{\mu} \rightarrow \nu_{\mu}}^{\min }
$$

Let us consider now the case $\left|U_{\mu 4}\right|^{2} \geq 1-a_{\mu}^{0}$, which implies

$$
P_{\nu_{\mu} \rightarrow \nu_{\mu}}^{\min }=\left(1-a_{\mu}^{0}\right)^{2}
$$

The solid curve in Fig.1 depicts the corresponding value of $R^{\text {min }}$ for the Kamiokande sub$\mathrm{GeV}$ events as a function of $\Delta m^{2}$. The shadowed horizontal band in Fig. I1 represents the $90 \%$ CL limits of the double ratio $R$ for the sub-GeV events determined in the Kamiokande experiment [11]. From Fig.1] one can see that, for $\Delta m^{2} \gtrsim 0.4 \mathrm{eV}^{2}$ and $\left|U_{\mu 4}\right|^{2} \geq 1-a_{\mu}^{0}$, the value of $R^{\text {min }}$ is bigger than the experimental upper limit for $R$.

With the argument presented above we cannot exclude large values of $\left|U_{\mu 4}\right|^{2}$ in the small interval $0.3 \mathrm{eV}^{2} \lesssim \Delta m^{2} \lesssim 0.4 \mathrm{eV}^{2}$. However, if the atmospheric neutrino anomaly is mainly due to a deficit of $\mu$-like events (as indicated by a comparison of the experimental data 111, 12, 13] with the existing calculations of the atmosperic neutrino fluxes [28], with the exception of the calculation presented in Ref. 229]), large values of $\left|U_{\mu 4}\right|^{2}$ are incompatible with the data for all values of $\Delta m^{2}$. This is connected with the fact that for large values of $\left|U_{\mu 4}\right|^{2}$ the coefficient $\left(1-\left|U_{\mu 4}\right|^{2}\right)^{2}$ of the survival probability $P_{\nu_{\mu} \rightarrow \nu_{\mu}}^{(1,2 ; 3)}$ in Eq.(15) is very small and it is not possible to explain the zenithal dependence observed in the Kamiokande multi-GeV data [11] (for example, at $\Delta m^{2}=0.3 \mathrm{eV}^{2}$ we have $\left.\left(1-\left|U_{\mu 4}\right|^{2}\right)^{2} \lesssim 0.06\right)$.

Thus, using the results of the reactor and accelerator disappearance experiments and taking into account the solar neutrino data and the atmospheric neutrino anomaly, we come to the conclusion that

$$
\left|U_{e 4}\right|^{2} \leq a_{e}^{0} \quad \text { and } \quad\left|U_{\mu 4}\right|^{2} \leq a_{\mu}^{0} .
$$

We will consider now $\nu_{\mu} \leftrightarrows \nu_{e}$ oscillations. From Eqs.(9) and (19) we have

$$
A_{\mu ; e}=4\left|U_{e 4}\right|^{2}\left|U_{\mu 4}\right|^{2} \leq 4 a_{e}^{0} a_{\mu}^{0} .
$$

Thus, the upper bound for the amplitude $A_{\mu ; e}$ is quadratic in the small quantities $a_{e}^{0}$, $a_{\mu}^{0}$, and $\nu_{\mu} \leftrightarrows \nu_{e}$ oscillations must be strongly suppressed if there is a neutrino mass hierarchy. In Fig.2 the limit (20) is presented as the curve passing through the circles. The $90 \%$ CL exclusion regions found in the $\bar{\nu}_{e}$ disappearance Bugey experiment and in the $\nu_{\mu} \rightarrow \nu_{e}$ appearance BNL E776 [30] and KARMEN [31] experiments are limited in Fig.2 by the dashed, dot-dashed and dot-dot-dashed curves, respectively. The shadowed region in Fig.2 is the region of the parameters $\Delta m^{2}$ and $A_{\mu ; e}$ which is allowed at $90 \%$ CL by the LSND experiment. It is seen from Fig.2 that the region allowed by LSND is inside of the regions that are forbidden by the results of all the other experiments. Thus, we come to the conclusion that a mass hierarchy of four neutrinos is not compatible with the results of all neutrino oscillation experiments. 


\section{Four massive neutrinos with non-hierarchial mass spectra}

If the neutrino masses satisfy the inequalities

$$
m_{1} \ll m_{2} \lesssim m_{3} \lesssim m_{4}
$$

with $\Delta m_{32}^{2}$ and $\Delta m_{43}^{2}$ relevant for the suppression of the solar $\nu_{e}$ 's and for the atmospheric neutrino anomaly, respectively, the short-baseline oscillation amplitudes are given by Eqs.(9) and (10) with the change $\left|U_{\alpha 4}\right|^{2} \rightarrow\left|U_{\alpha 1}\right|^{2}$. Arguments similar to those presented in Section 3 lead us to the conclusion that the mass spectrum (21) is disfavored by the experimental data. In a similar manner one can demonstrate that all possible schemes with mass spectra in which three masses are clustered and one mass is separated from the cluster by the $\sim 1 \mathrm{eV}$ gap needed for the explanation of the LSND data are not compatible with the results of all neutrino oscillation experiments.

Now we are left only with two possible neutrino mass spectra in which the four neutrino masses appear in two pairs separated by $\sim 1 \mathrm{eV}$ :

$$
\underbrace{\overbrace{m_{1}<m_{2}}^{\text {atm }} \ll \overbrace{m_{3}<m_{4}}^{\text {solar }}}_{\text {LSND }} \text { and }
$$

$$
\underbrace{\overbrace{m_{1}<m_{2}}^{\text {solar }} \ll \overbrace{m_{3}<m_{4}}^{\text {atm }}}_{\text {LSND }} .
$$

The possible effects of these neutrino mass spectra have been discussed in Refs. [32, 15]. We will show now that the schemes with the spectra (A) and (B) are compatible with the results of all neutrino oscillation experiments. Let us define the quantities

$$
c_{\alpha} \equiv \sum_{i=1,2}\left|U_{\alpha i}\right|^{2} \quad(\alpha=e, \mu)
$$

For both schemes (A) and (B) the amplitude $B_{\alpha ; \alpha}$ is given by (see Eq.(6) with $n=4$ and $r=2$ )

$$
B_{\alpha ; \alpha}=4 c_{\alpha}\left(1-c_{\alpha}\right)
$$

From the results of reactor and accelerator disappearance experiments it follows that the parameters $c_{\alpha}$ must satisfy one of the two inequalities

$$
c_{\alpha} \leq a_{\alpha}^{0} \quad \text { or } \quad c_{\alpha} \geq 1-a_{\alpha}^{0} \quad(\alpha=e, \mu),
$$

where $a_{\alpha}^{0}$ is given by Eq.(13).

Let us first consider the scheme (A). For the probabilities of solar $\nu_{e}$ 's and atmospheric $\nu_{\mu}$ 's to survive we have

$$
P_{\nu_{e} \rightarrow \nu_{e}}^{\odot}=\sum_{i=1,2}\left|U_{e i}\right|^{4}+\left(1-c_{e}\right)^{2} P_{\nu_{e} \rightarrow \nu_{e}}^{(3 ; 4)}
$$

and

$$
P_{\nu_{\mu} \rightarrow \nu_{\mu}}^{\mathrm{atm}}=\left(1-c_{\mu}\right)^{2}+c_{\mu}^{2} P_{\nu_{\mu} \rightarrow \nu_{\mu}}^{(1 ; 2)}
$$


If $c_{e} \geq 1-a_{e}^{0}$, from Eq.(26) it follows that the survival probability of solar $\nu_{e}$ 's, $P_{\nu_{e} \rightarrow \nu_{e}}^{\odot}$, practically does not depend on the neutrino energy and

$$
P_{\nu_{e} \rightarrow \nu_{e}}^{\odot} \gtrsim 0.5 \text {. }
$$

This is disfavored by the solar neutrino data [33]. If $c_{\mu} \leq a_{\mu}^{0}$, from Eq. (27) we have

$$
P_{\nu_{\mu} \rightarrow \nu_{\mu}}^{\mathrm{atm}} \geq\left(1-a_{\mu}^{0}\right)^{2},
$$

which is not compatible with the Kamiokande atmospheric neutrino data for the reasons discussed in Section 3. Thus, in order to accomodate the solar neutrino data and the atmospheric neutrino anomaly, from the four possibilities (25) we must choose

$$
c_{e} \leq a_{e}^{0} \quad \text { and } \quad c_{\mu} \geq 1-a_{\mu}^{0} .
$$

Let us consider now $\nu_{\mu} \leftrightarrows \nu_{e}$ oscillations. Using the Cauchy-Schwarz inequality, from Eq.(四) (with $n=4$ and $r=2$ ) and Eq.(23), for both schemes (A) and (B) we find

$$
A_{\mu ; e}=4\left|\sum_{i=1,2} U_{e i} U_{\mu i}^{*}\right|^{2} \leq 4 c_{e} c_{\mu} .
$$

From Eqs.(30) and (31) it follows that the upper bound for $A_{\mu ; e}$ is linear in the small quantity $a_{e}^{0}$. Since $a_{e}^{0} \gtrsim 5 \times 10^{-3}$ for all values of $\Delta m^{2}$, in the case of the scheme (A) the limit (31) is compatible with the results of the LSND experiment.

In the case of the scheme (B), the solar neutrino problem and the atmospheric neutrino anomaly can be explained by neutrino oscillations only if

$$
c_{e} \geq 1-a_{e}^{0} \quad \text { and } \quad c_{\mu} \leq a_{\mu}^{0} .
$$

From Eqs.(31) and (32) it follows that the scheme (B) is also compatible with the results of the LSND experiment.

The schemes (A) and (B) lead to different consequences for the experiments on the mesurement of the neutrino mass through the investigation of the end-point part of the ${ }^{3} \mathrm{H} \beta$-spectrum and for the experiments on the search for neutrinoless double- $\beta$ decay $\left((\beta \beta)_{0 \nu}\right)$. In fact, for the whole range of $\Delta m^{2}$ considered here we have

$$
\begin{aligned}
& \text { (A) } \sum_{i=3,4}\left|U_{e i}\right|^{2} \geq 1-a_{e}^{0}, \\
& \text { (B) } \sum_{i=3,4}\left|U_{e i}\right|^{2} \leq a_{e}^{0} .
\end{aligned}
$$

From Eq.(33) it follows that in the case of the scheme (A) the neutrino mass that enters in the usual expression for the $\beta$ spectrum of ${ }^{3} \mathrm{H}$ decay (see Ref. [34]) is approximately equal to the "LSND mass" $m_{4}$ :

$$
m_{\nu}\left({ }^{3} \mathrm{H}\right) \simeq m_{4} .
$$

If the scheme (B) is realized in nature and $m_{1}, m_{2}$ are very small, the mass measured in ${ }^{3} \mathrm{H}$ experiments is at least two order of magnitude smaller than $m_{4}$. 
If massive neutrinos are Majorana particles, $(\beta \beta)_{0 \nu}$ decay is possible. In the scheme (A), the effective neutrino "mass" $|\langle m\rangle|=\left|\sum_{i=1}^{n} U_{e i}^{2} m_{i}\right|$ that is measured in $(\beta \beta)_{0 \nu}$ decay is given by

$$
|\langle m\rangle| \simeq\left|\sum_{i=3,4} U_{e i}^{2}\right| m_{4}
$$

We have

$$
|\langle m\rangle| \simeq m_{4} \sqrt{1-4\left|U_{e 4}\right|^{2}\left(1-\left|U_{e 4}\right|^{2}\right) \sin ^{2} \phi},
$$

where $\phi$ is the difference of the phases of $U_{e 3}$ and $U_{e 4}$. Depending on the value of the phase $\phi$, the quantity $|\langle m\rangle|$ has a value in the range

$$
\left.|2| U_{e 4}\right|^{2}-1\left|m_{4} \lesssim\right|\langle m\rangle \mid \lesssim m_{4}
$$

The upper and lower bounds in Eq.(38) correspond, respectively, to the cases of equal and opposite CP parities of $\nu_{3}$ and $\nu_{4}$ (see Ref. [15]). From Eq.(34) it follows that, if $m_{1}$ and $m_{2}$ are very small, in the case of the mass spectrum (B) the expected value of $|\langle m\rangle|$ is very small.

Thus, the experiments on the investigation of the effects of neutrino masses with the measurement of the end-point part of the $\beta$-spectrum of ${ }^{3} \mathrm{H}$ and with the search for $(\beta \beta)_{0 \nu}$ decay could allow to distinguish between the possibilities (A) or (B) for the neutrino mass spectrum.

\section{Conclusions}

In this paper we have discussed the possible form of the neutrino mass spectrum that can be inferred from the results of all neutrino oscillation experiments, including the solar and atmospheric neutrino experiments. In this investigation we assumed only that one neutrino mass squared difference is relevant in short-baseline oscillation experiments. We have argued that it is unlikely that all data can be fitted with three neutrinos, particularly if one includes the Kamiokande multi-GeV results.

The experimental indications in favor of neutrino mixing coming from the results of the solar and atmospheric neutrino experiments and of the LSND experiment imply that there are (at least) three different scales of $\Delta m^{2}$ 's, about $10^{-5} \mathrm{eV}^{2}, 10^{-2} \mathrm{eV}^{2}$ and $1 \mathrm{eV}^{2}$. We have considered all the possible schemes with four massive neutrinos which provide these three scales of $\Delta m^{2}$ 's.

We have shown that the results of the LSND experiment are not compatible with the limits obtained by all the other neutrino oscillation experiments in the case of a neutrino mass hierarchy $\left(m_{1} \ll m_{2} \ll m_{3} \ll m_{4}\right)$ and in the cases of neutrino mass spectra in which three masses are clustered in a group and one mass is separated from the cluster with a mass difference of the order of $1 \mathrm{eV}$, which corresponds to the range of $\Delta m^{2}$ relevant for the oscillations observed in the LSND experiment. 
We have also shown that only two possible spectra of neutrino masses, (A) and (B) (see (22)), with two pairs of close masses separated by a mass difference of the order of $1 \mathrm{eV}$ are compatible with the results of all neutrino oscillation experiments. If the neutrino mass spectrum (A) is realized in nature, the neutrino mass that is measured in ${ }^{3} \mathrm{H} \beta$-decay experiments coincide with the "LSND mass". If the massive neutrinos are Majorana particles, in the case of scheme $(\mathrm{A})$, the experiments on the search for $(\beta \beta)_{0 \nu}$ decay have good chances to obtain a positive result.

Finally, we want to remark that, if the experimental indications in favor of neutrino oscillations are confirmed, the neutrino mass spectrum is very different from the mass spectra of quarks and charged leptons. This is, however, not so astonishing, because at least four neutrinos, one of which is sterile, are necessary in order to explain the results of all neutrino oscillation experiments.

\section{Note Added}

After this work was finished and reported at the Neutrino '96 Conference in Helsinki, the preprint TMUP-HEL-9605 by N. Okada and O Yasuda appeared (hepph/9606411). Some of our results are also contained in this paper.

\section{Acknowledments}

We would like to thank J. Bernabeu, G. Conforto, F. Martelli, L. Mikaelian and S. Petcov for useful discussions. S.M.B. would like to express his deep gratitude to Prof. H. Pietschmann for the kind hospitality at the Institute for Theoretical Physics of the University of Vienna.

\section{References}

[1] R.N. Mohapatra and P.B. Pal, "Massive Neutrinos in Physics and Astrophysics", World Scientific Lecture Notes in Physics-Vol. 41, World Scientific, Singapore, 1991.

[2] B.T. Cleveland et al., Nucl. Phys. B (Proc. Suppl.) 38, 47 (1995).

[3] K.S. Hirata et al., Phys. Rev. D 44, 2241 (1991).

[4] GALLEX Coll., Phys. Lett. B 357, 237 (1995).

[5] J.N. Abdurashitov et al., Phys. Lett. B 328, 234 (1994).

[6] J.N. Bahcall and M.H. Pinsonneault, Rev. Mod. Phys. 67, 781 (1995); S. TurckChièze et al., Phys. Rep. 230, 57 (1993); V. Castellani et al., preprint INFNFE-10-96 (e-Print Archive: astro-ph/9606180); A. Dar and G. Shaviv, Nucl. Phys. B (Proc. Suppl.) 48, 335 (1996).

[7] V. Castellani et al., Astron. Astrophys. 271, 601 (1993); S.A. Bludman et al., Phys. Rev. D 49, 3622 (1994); V. Berezinsky, Comm. Nucl. Part. Phys. 21, 249 (1994); J.N. Bahcall, Phys. Lett. B 338, 276 (1994). 
[8] S.P. Mikheyev and A.Yu. Smirnov, Yad. Fiz. 42, 1441 (1985) [Sov. J. Nucl. Phys. 42, 913 (1985)]; Il Nuovo Cimento C 9, 17 (1986); L. Wolfenstein, Phys. Rev. D 17, 2369 (1978); Phys. Rev. D 20, 2634 (1979).

[9] GALleX Coll., Phys. Lett. B 285, 390 (1992); X. Shi, D.N. Schramm and J.N. Bahcall, Phys. Rev. Lett. 69, 717 (1992); P.I. Krastev and S.T. Petcov, Phys. Lett. B 299, 99 (1993); N. Hata and P.G. Langacker, Phys. Rev. 50, 632 (1994); G.L. Fogli and E. Lisi, Astropart. Phys. 2, 91 (1994); G. Fiorentini et al., Phys. Rev. D 49, 6298 (1994); L.M. Krauss, E. Gates and M. White, Phys. Rev. D 51, 2631 (1995).

[10] V. Barger, R.J.N. Phillips, and K. Whisnant, Phys. Rev. Lett. 69, 3135 (1992); P.I. Krastev and S.T. Petcov, Phys. Rev. Lett. 72, 1960 (1994).

[11] Y. Fukuda et al., Phys. Lett. B 335, 237 (1994).

[12] R. Becker-Szendy et al., Nucl. Phys. B (Proc. Suppl.) 38, 331 (1995).

[13] E.A. Peterson, Talk presented at the XVII International Conference on Neutrino Physics and Astrophysics, Helsinki, June 1996.

[14] C. Athanassopoulos et al., Phys. Rev. Lett. 75, 2650 (1995); preprint LA-UR-96-1326 (e-Print Archive: nucl-ex/9605001).

[15] S.M. Bilenky, C. Giunti, C.W. Kim and S.T. Petcov, preprint SISSA 35/96/EP (e-Print Archive: hep-ph/9604364).

[16] A. De Rujula et al., Nucl. Phys. B 168, 54 (1980); V. Barger and K. Whisnant, Phys. Lett. B 209, 365 (1988); S.M. Bilenky, M. Fabbrichesi and S.T. Petcov, Phys. Lett. B 276, 223 (1992); G.L. Fogli, E. Lisi and G. Scioscia, Phys. Rev. D 52, 5334 (1995); H. Minakata, Phys. Lett. B 356, 61 (1995); Phys. Rev. D 52, 6630 (1995); S. Goswami, K. Kar and A. Raychaudhuri, preprint CUPP-95/3 (e-Print Archive: hep-ph/9505395); K.S. Babu, J.C. Pati and F. Wilczek, Phys. Lett. B 359, 351 (1995).

[17] S.M. Bilenky, A. Bottino, C. Giunti and C.W. Kim, Phys. Lett. B 356, 273 (1995); Phys. Rev. D 54 (1996), in press (e-Print Archive: hep-ph/9602216).

[18] C.Y. Cardall and G.M. Fuller, Phys. Rev. D 53, 4421 (1996); O. Yasuda and H. Minakata, preprint TMUP-HEL-9604 (hep-ph/9602386).

[19] B. Achkar et al., Nucl. Phys. B 434, 503 (1995).

[20] F. Dydak et al., Phys. Lett. B 134, 281 (1984).

[21] Y. Suzuki, Talk presented at the XVII International Conference on Neutrino Physics and Astrophysics, Helsinki, June 1996.

[22] D. Macina, Nucl. Phys. B (Proc. Suppl.) 48, 183 (1996).

[23] M. Laveder, Nucl. Phys. B (Proc. Suppl.) 48, 188 (1996). 
[24] R.A. Sidwell, Talk presented at the XVII International Conference on Neutrino Physics and Astrophysics, Helsinki, June 1996.

[25] S.T. Petcov and A.Yu. Smirnov, Phys. Lett. B 322, 109 (1994); D.O. Caldwell and R.N. Mohapatra, Phys. Lett. B 354, 371 (1995); G. Raffelt and J. Silk, Phys. Lett. B 366, 429 (1996).

[26] I.E. Stockdale et al., Phys. Rev. Lett. 52, 1384 (1984).

[27] X. Shi and D.N. Schramm, Phys. Lett. B 283, 305 (1992).

[28] G. Barr, T.K. Gaisser and T. Stanev, Phys. Rev. D 39, 3532 (1989); M. Honda et al., Phys. Lett. B 248, 193 (1990).

[29] E.V. Bugaev and V.A. Naumov, Phys. Lett. B 232, 391 (1989).

[30] L. Borodovsky et al., Phys. Rev. Lett. 68, 274 (1992).

[31] J. Kleinfeller, Nucl. Phys. B (Proc. Suppl.) 48, 207 (1996).

[32] J.T. Peltoniemi and J.W.F. Valle, Nucl. Phys. B 406, 409 (1993); D.O. Caldwell and R.N. Mohapatra, Phys. Rev. D 50, 3477 (1994); Z. Berezhiani and R.N. Mohapatra, Phys. Rev. D 52, 6607 (1995); J.R. Primack et al., Phys. Rev. Lett. 74, 2160 (1995); E. Ma and P. Roy, Phys. Rev. D 52, R4780 (1995); R Foot and R.R. Volkas, Phys. Rev. D 52, 6595 (1995); E.J. Chun, A.S. Joshipura and A.Yu. Smirnov, Phys. Lett. B 357, 608 (1995); J.J. Gomez-Cadenas and M.C. Gonzalez-Garcia, preprint CERNTH/95-80 (e-Print Archive: hep-ph/9504246); S. Goswami, preprint CUPP-95/4 (ePrint Archive: hep-ph/9507212); A.Yu. Smirnov and M. Tanimoto, preprint EHU96-04 (e-Print Archive: hep-ph/9604370); E. Ma, preprint UCRHEP-T-166 (e-Print Archive: hep-ph/9606360).

[33] P.I. Krastev and S.T. Petcov, Phys. Rev. D 53, 1665 (1996).

[34] R.G.H. Robertson and D.A. Knapp, Ann. Rev. Nucl. Part. Sci. 38, 185 (1988). 


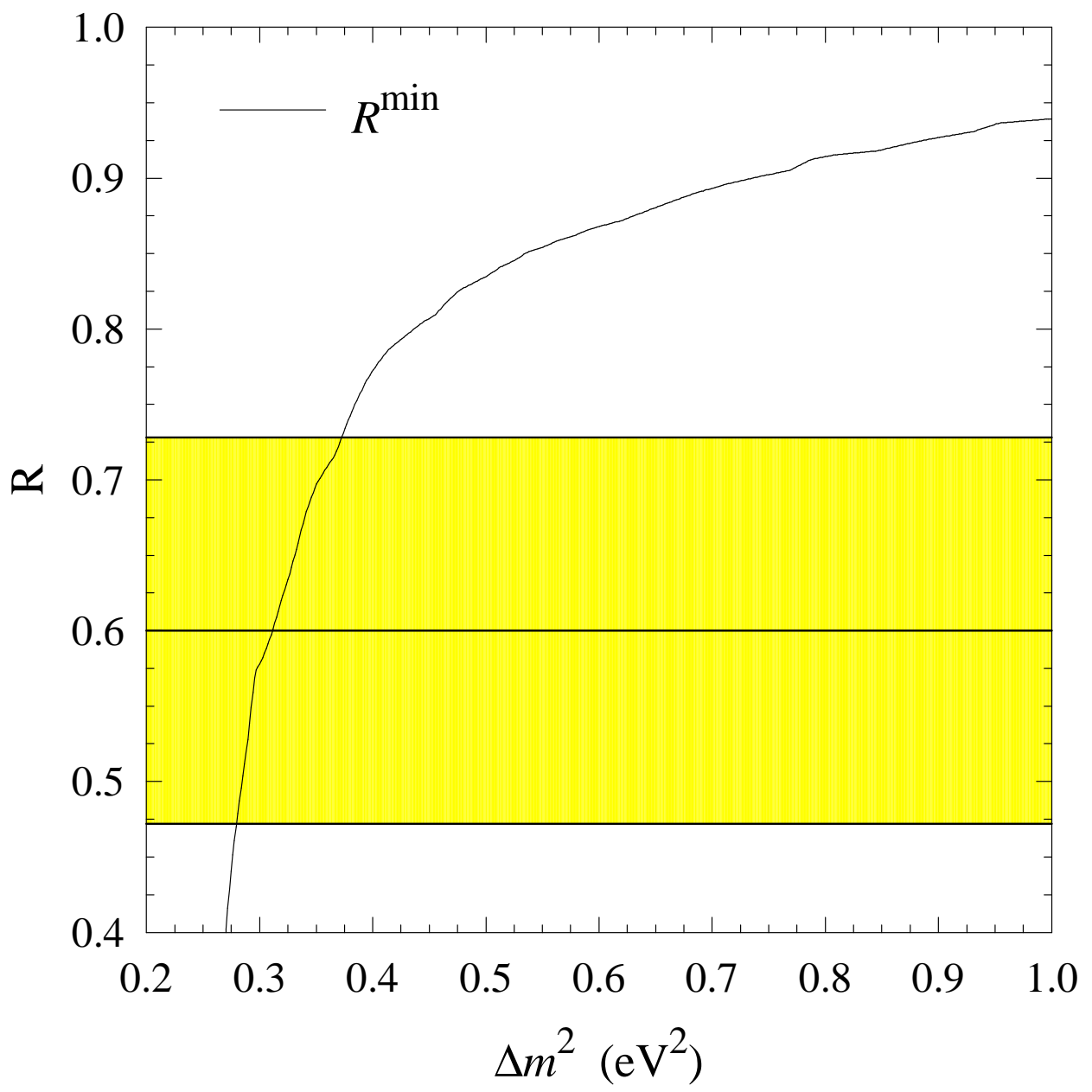

Figure 1: Value of $R^{\text {min }}$ for the Kamiokande sub-GeV data (see Eq.(17)) as a function of $\Delta m^{2}$. The shadowed horizontal band represents the $90 \%$ CL limits of the double ratio $R$ for the sub-GeV events determined in the Kamiokande experiment [11]. 


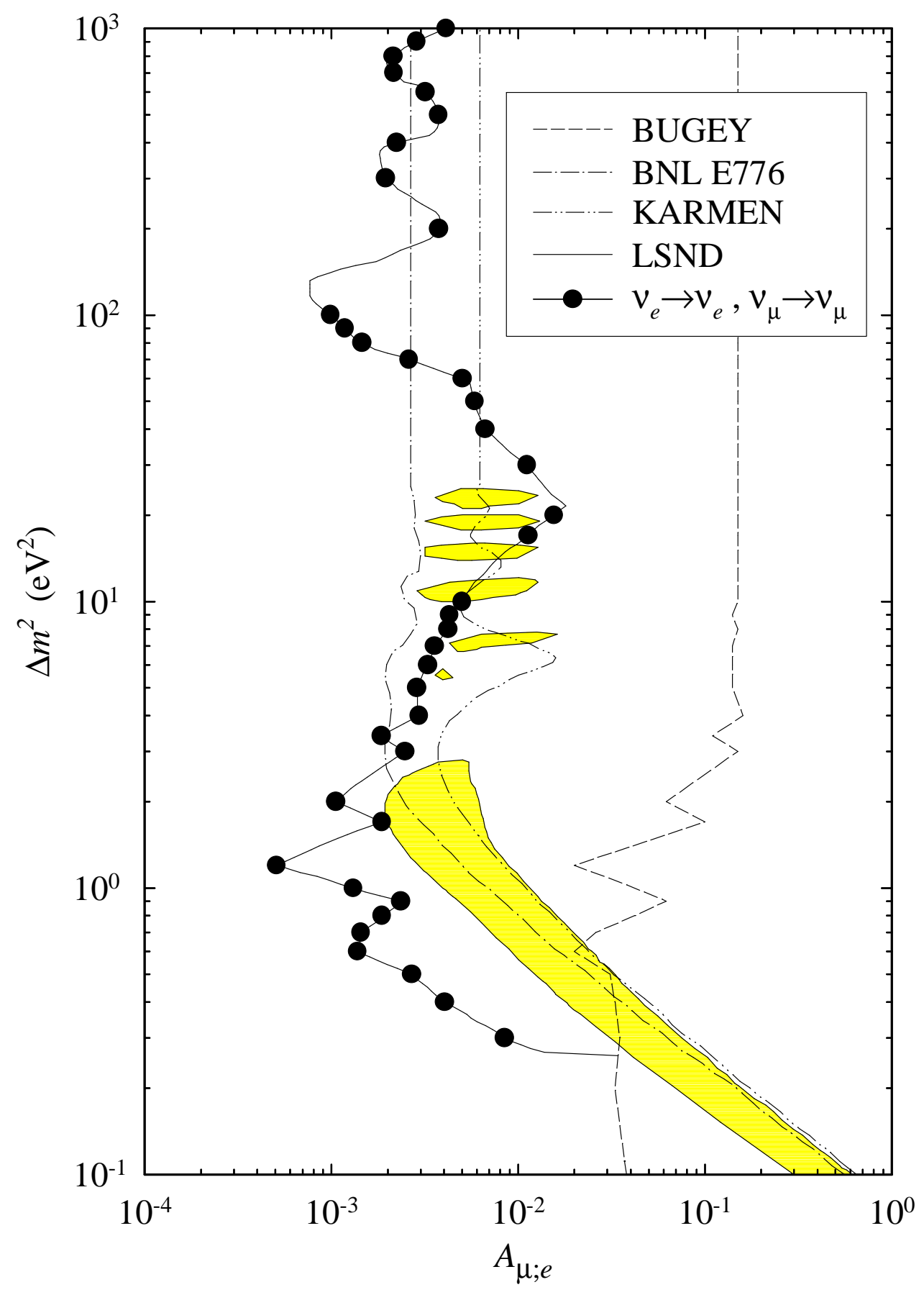

Figure 2: Exclusion regions al 90\% CL in the $A_{\mu ; e}-\Delta m^{2}$ plane for small $\left|U_{e 4}\right|^{2}$ and $\left|U_{\mu 4}\right|^{2}$ in the model with mixing of four neutrinos and a mass hierarchy discussed in Section 3. The regions excluded by the BNL E776 and KARMEN $\nu_{\mu} \rightarrow \nu_{e}$ appearance experiments are bounded by the dash-dotted and dash-dot-dotted curves, respectively. The dashed line represents the results of the Bugey experiment. The curve passing through the circles is obtained from the results of the Bugey, CDHS and CCFR84 experiments using Eq.(20). The region allowed by the LSND experiment is shown as the shadowed region limited by the two solid curves. 